Voix et Images

voixetimages

\title{
Bibliographie de Michel Beaulieu
}

\section{Frédéric Rondeau}

Volume 33, numéro 2 (98), hiver 2008

Michel Beaulieu

URI : https://id.erudit.org/iderudit/018270ar

DOI : https://doi.org/10.7202/018270ar

Aller au sommaire du numéro

\section{Éditeur(s)}

Université du Québec à Montréal

\section{ISSN}

0318-9201 (imprimé)

1705-933X (numérique)

Découvrir la revue

\section{Citer ce document}

Rondeau, F. (2008). Bibliographie de Michel Beaulieu. Voix et Images, 33(2),

97-112. https://doi.org/10.7202/018270ar d'utilisation que vous pouvez consulter en ligne.

https://apropos.erudit.org/fr/usagers/politique-dutilisation/ 


\title{
B I B L I O G R A P H IE D E M I C HEL B E A U L IE U
}

$+++$

\author{
FRÉDÉRIC RONDEAU \\ Université McGill
}

Les poèmes et les textes en prose qui ont été rassemblés en volume ainsi que les articles de critique rédigés par Michel Beaulieu n'ont pas été recensés dans cette bibliographie. Pour la liste exhaustive des publications jusqu'à 1985, on peut consulter la précieuse biobibliographie analytique établie par Lise-Anne Bélanger ${ }^{1}$. Afin de faciliter leur repérage, les références sont classées par ordre chronologique.

\section{E U V R E S}

\section{1. Recueils de poésie}

+ Pour chanter dans les chaînes, Montréal, Éditions la Québécoise, 1964, [n.p.]. (Repris partiellement dans Desseins. Poèmes, 1961-1966, 1980.)

+ Le pain quotidien (avec sept dessins à l'encre de Jean McEwen), Montréal, Éditions Estérel, 1965, 96 p. (Repris partiellement dans Desseins. Poèmes, 1961-1966, 1980.)

+ «Ballades... et satires», dans Trois, recueil publié conjointement avec Nicole Brossard et Micheline de Jordy, Montréal, les Presses de l'A.G.E.U.M., cahier nº 12, 1965, 91 p. (Repris partiellement dans Desseins. Poèmes, 1961-1966, 1980.)

+ Apatride (Gestes II) (avec des eaux-fortes de Roland Pichet), Montréal, Éditions Estérel, 1966, [n.p.]. (Repris partiellement dans Desseins. Poèmes, 1961-1966, 1980.)

+ Mère (avec neuf bois gravés de Roland Pichet), Montréal, Éditions Estérel, 1966, [n.p.]. (Repris partiellement dans Desseins. Poèmes, 1961-1966, 1980.)

+ Érosions, Montréal, Éditions Estérel, 1967, 57 p. (Repris partiellement dans Desseins. Poèmes, 1961-1966, 1980.)

+ 0: 00, Montréal, Éditions Estérel, 1969, 80 p. (Repris partiellement dans Charmes de la fureur, 1970 et dans Études françaises, vol. IV, no 4, novembre 1968, p. 395-402.)

+ Charmes de la fureur, Montréal, Éditions du Jour, coll. «Les poètes du jour », 1970, 75 p.

+ Sous-jacences (avec des images de Roland Pichet), Montréal, R. Perreault, 1970, [n.p.].

+ Paysage, précédé de Adn, Montréal, Éditions du Jour, coll. «Les poètes du jour», 1971, 100 p.

+ Pulsions, Montréal, l’Hexagone, 1973, 58 p.

$$
+++
$$

1 Lise-Anne Bélanger, «Bio-bibliographie de Michel Beaulieu, écrivain (1941-1985)», mémoire de maîtrise, Montréal, Université de Montréal, 1987, 378 f. 
+ Variables, Montréal, Les Presses de l'Université de Montréal, coll. «Prix de la revue Études françaises", 1973, $110 \mathrm{p}$.

+ FM. Lettres des saisons III, Montréal, Éditions du Noroît, 1975, [n.p.].

+ Le Flying Dutchman, Montréal, Éditions Cul Q, coll. «Mium-mium», n 9, 1976, [n.p.].

+ Suite québécoise. Blues pour un piquet de clôture (avec Nicole Brossard, Roch Carrier, Paul Chamberland, Robert Charlebois, Yvon Deschamps, Georges Dor, Raoul Duguay, Jean Royer, Gilles Vigneault et onze images de Roland Pichet), Montréal, Éditions du Songe/Iconia, 1976, [n.p.].

+ Anecdotes (avec huit encres de Louise Thibault), Montréal, Éditions du Noroît, 1977, 63 p.

+ Le cercle de justice, Montréal, l'Hexagone, 1977, 95 p.

+ Indicatif présent (avec quatre encres de Carol Dunlop), Montréal, Éditions Estérel, 1977, [n.p.].

+ L'octobre, suivi de Dérives, Montréal, l'Hexagone, 1977, 78 p.

+ Comment ça va?, Montréal, Éditions Cul Q, coll. «Mium-mium», nº 22, 1978, 27 p.

+ Familles, Montréal, Éditions Estérel, 1978, [n.p.].

+ Oratorio pour un prophète, Montréal, Éditions Estérel, 1978, [n.p.].

+ Amorces, Montréal, Éditions Estérel, 1979, [n.p.].

+ Civilités, Montréal, Éditions Estêrel, 1979, [n.p.].

+ Fléchettes, Montréal, Éditions Minimales, 1979, [n.p.].

+ Oracle des ombres (avec illustrations de Sylvie Melançon), Montréal, Éditions du Noroît, 1979, [n.p.].

+ Rémission du corps énamouré, Montréal, Éditions du Mouton noir, 1979, [n.p.].

+ Zoo d'espèces, Montréal, Éditions du Mouton noir, 1979, [n.p.].

+ Desseins. Poèmes, 1961-1966, Montréal, l'Hexagone, coll. «Rétrospectives», 1980, 246 p.

+ Sept fois tournée la langue effleure, Montréal, Éditions Minimales, 1980, [n.p.].

+ Visages, Montréal, Éditions du Noroît, 1981, 134 p.

+ Hibernation, Montréal, Éditions du Mouton noir, 1982, [n.p.].

+ Images du temps (avec six lithographies et six gaufrures de Gilles Boisvert), Montréal, Éditions du Noroît, 1983, 1 emboîtage, 14 cahiers.

+ Natalités (avec cinq eaux-fortes de Monique Voyer), Magog, Éditions Sylvédite, 1984, 1 emboîtage, [n.p.].

+ Kaléidoscope ou Les aléas du corps grave, Montréal, Éditions du Noroît, 1984, 149 p.

+ Quadrature (avec une sérigraphie de Roland Giguère), Montréal, Éditions du Silence, 1989, [n.p.].

+ Vu, Montréal/Pantin, Éditions du Noroît/Castor astral, 1989, 121 p.

+ Indicatif présent et autres poèmes, Montréal, Éditions du Noroît, 1993, 120 p.

+ Fuseaux. Poèmes choisis, Saint-Hippolyte, Éditions du Noroît, coll. «Ovale», 1996, 116 p.

+ Trivialités, Montréal, Éditions du Noroît, 2001, 114 p.

\section{I . 2. R o m a n s}

+ Je tourne en rond mais c'est autour de toi, Montréal, Éditions du Jour, coll. «Les romanciers du jour", 1969, $179 \mathrm{p}$.

+ La représentation, Montréal, Éditions du Jour, coll. «Les romanciers du jour», 1972, 198 p.

+ Sylvie Stone, Montréal, Éditions du Jour, coll. «Les romanciers du jour», 1974, 177 p.

+ Je tourne en rond mais c'est autour de toi, 2e édition, Montréal, Quinze, coll. «Présence», 1980, $183 \mathrm{p}$. 
+ La représentation, 2e édition, Montréal, Quinze, coll. «Présence», 1980, 213 p.

+ Sylvie Stone, 2e édition, Montréal, Quinze, coll. «Présence», 1980, 182 p.

\section{I . 3. R é c it}

+ X, Montréal, Chez l'Obscène Nyctalope, 1968, [32] p.

\section{4. Monographie}

+ P. V. Beaulieu, textes de Jacques Brault et de Michel Beaulieu, La Prairie, Éditions Marcel Broquet, coll. «Signatures», 1981, 106 p.

\section{I.5. Théâtre (texte inédit)}

+ Jeudi soir en pleine face, 1975. Coproduite avec le théâtre de la Manufacture et créée par le théâtre de Quat'sous du 29 janvier au 21 février 1976.

\section{I.6. Poèmes et proses (non parus en recueil)}

+ «Danse d'automne», Journal Brébeuf, vol. XXVIII, n 2, 23 octobre 1959, p. 1.

+ «Poèmes du bonheur de Danie (fragments)», Le Quartier latin, vol. XLVI, nº 50, 9 avril 1964, supplément (I), p. 2.

+ «Coordonnées», La Barre du jour, vol. I, nº 1, janvier-février 1965, p. 11-14.

+ «Manifeste [I] », Le Quartier latin, vol. XLVII, no 45, 18 mars 1965, supplément, p. 6.

+ "Érosions», La Barre du jour, n 7, été 1966, p. 24-27.

+ «[moi qui dérive selon l'ordre]», La Canadienne, n² 29, octobre 1966, p. 33.

+ «Le quoi de quoi», Quoi, vol. I, nº 1, janvier-février 1967, p. 3-6.

+ «Huit équinoxes réfractaires», Quoi, vol. I, no 1, janvier-février 1967, p. 29-33.

+ "Pour un essai de justification passive», Quoi, vol. I, nº 2, printemps-été 1967, p. 7-10.

+ «Poèmes de l'amour en sursis», Liberté, vol. IX, nº 6 (n 54), novembre-décembre 1967, p. 55.

+ «Texte», La Barre du jour, nos 17-18-19, janvier-août 1969, p. 271-273.

+ «Lettre ouverte à Léo Bonneville», Le Devoir, 26 avril 1969, p. 2, 4.

+ «Réponse au questionnaire de Marcel Proust», Victor-Lévy Beaulieu (dir.), Les écrivains québécois jouent le jeu, Montréal, Éditions du Jour, 1970, p. 23-26.

+ «Une écriture de toute semaine», Littérature du Québec. Poésie actuelle, Guy Robert (dir.), Montréal, Librairie Déom, 1970, p. 344-351.

+ «Suffit», L'Illettré, vol. I, no 1, janvier 1970, [n.p.].

+ «Deux poèmes», L'Illettré, vol. I, no 3, mars 1970, [n.p.].

+ «Une jeune femme, un jour...», La Presse, 23 mai 1970, p. 29.

+ «Es», La Barre du jour, vol. V, n² 24, été 1970, p. 27-35.

+ "Quelques mots d'un jour ensoleillé», L'Illettré, vol. I, nº 4, été 1970, p. 1.

+ «Lettre ouverte», La Barre du jour, no 26, octobre 1970, p. 50.

+ «Octobre un soir», La Barre du jour, n 28, mai 1971, p. 43-61.

+ «La femme qui marche», La Barre du jour, n²9, été 1971, p. 54-84.

+ «Salut!», Le Devoir, «supplément littéraire», 30 octobre 1971, p. 38.

+ «Science-friction», Hobo-Québec, nos 5-7, été 1973, p. 12.

+ «Ā bout portant», Hobo-Québec, nºs 5-7, juin-juillet-août 1973, p. 21.

+ «À bout portant», Hobo-Québec, septembre 1973, p. 3.

+ «Dédales», Hobo-Québec, nos 12-13, décembre 1973, p. 15. 
+ «Une femme qui marche», Hobo-Québec, nos 12-13, décembre 1973, p. 16.

+ «Nouveaux poèmes», Hobo-Québec, n 31, septembre-décembre 1976, p. 19.

+ «Nouveaux poèmes», La Barre du jour, no 52, septembre-octobre 1976, p. 52-65. (Poèmes repris dans Oracle des ombres (avec illustrations de Sylvie Melançon), Montréal, Éditions du Noroît, 1979, [n.p.], sauf : «l'ordre des mots»)

+ "Veillée d'armes», La Barre du jour, n 55, mars-avril 1977, p. 44-56.

+ «Têtes chercheuses», La Nouvelle Barre du jour, n 60, novembre 1977, p. 40-51.

+ «Effraction», La Nouvelle Barre du jour, nos 68-69, septembre 1978, p. 127-128.

+ «Encres», La Nouvelle Barre du jour, n 82, octobre 1979, p. 26-35.

+ «1979.3.1-1976.3.6.», La Nouvelle Barre du jour, nos 100-101, mars 1981, p. 15-18.

+ «Intermission», suivi de "Variantes», Liberté, vol. XXV, n 1 (nº 145), février 1983, p. 11-12.

+ «Premier amour», Estuaire, nº 27, printemps 1983, p. 53-64.

+ «[Deux poèmes]», Estuaire, nos 32-33, été-automne 1984, p. 12-13.

+ «Morsures», Dalhousie French Studies, «La poésie québécoise depuis 1975 », 1985, p. 148-150.

\section{I.7. Pièces radiophoniques (textes inédits)}

+ "Un coup de pouce» et "L'édition spéciale», Premières, Montréal, Radio-Canada, 21 novembre 1974.

+ «L'explication» et «Paul, pas Paul», Premières, Montréal, Radio-Canada, 5 mars 1976.

+ «Si seulement...» et "Le voyageur», Premières, Montréal, Radio-Canada, 25 juin 1976.

+ «Une salade aux noix et aux échalotes» et «Vois-tu, vois-tu», Premières, Montréal, RadioCanada, 17 septembre 1976.

+ «Le dernier passage», La feuillaison, Montréal, Radio-Canada, 5 décembre 1976.

+ «Une obsession » et "On va-t-y finir ça ailleurs », Premières, Montréal, Radio-Canada, $1^{\text {er }}$ avril 1977.

+ «Corrida», L'atelier des inédits, Montréal, Radio-Canada, 23 avril 1977.

+ «Quelle belle journée pour mourir», Premières, Montréal, Radio-Canada, 11 novembre 1977.

+ «Une mort comme une autre», Premières, Montréal, Radio-Canada, 14 novembre 1977.

+ «Sais-tu seulement ce que t’as tuée?, Premières, Montréal, Radio-Canada, 25 novembre 1977.

+ «On ne joue pas au bolo», Premières, Montréal, Radio-Canada, 16 juin 1978.

\section{I.8. Enregistrement sonore}

+ Poèmes choisis, lus par Pierre Nepveu, musique de Violaine Corradi, Montréal, Éditions du Noroît, 1995, cassette analogique.

\section{9. Com mu nications}

+ «Une question de réponses», Liberté, vol. IX, nº 6 (n 54), novembre-décembre 1967, p. 74. Question posée «Qu'appelleriez-vous l'érotisme? » Répondants: Henri Jones, Jacques Ferron, Jean-Louis Major, François Piazza et Michel Beaulieu.

+ «Roman des Amériques», Rencontre internationale des écrivains tenue du 6 au 13 septembre 1973, Liberté, vol. XV, nº 6 (n 90), novembre-décembre 1973, p. 209-253.

\section{10. Entretiens}

+ ROCHE, Sylvie, "Je fais l'expérience du loup solitaire... », Le Quartier latin, "supplément», vol. XLVII, n 14, 29 octobre 1964, p. 1. 
+ LATOUR, Luc, «Interview de Luc Latour avec Michel Beaulieu», Le Quartier latin, vol. XLIX, n 38, 23 février 1967, p. 1-3.

+ GODIN, Gérald, «Le plus jeune éditeur du monde», Maclean, vol. VII, n 8, août 1967, p. 46.

+ BASILE, Jean, «Michel Beaulieu ou le risque de l'avant-garde», Le Devoir, 18 novembre 1967, p. 14.

+ MAJOR, André, "Sous le soleil des écrivains résistants", Le Devoir, 4 septembre 1969, p. 10.

+ BÉDARD, Colette, «Pas de vacances pour les idoles», L'Illettré, été 1970, [n.p.].

+ MARCOTTE, Gilles, "La Louisiane et le fait français», Perspectives, 24 mars 1973, p. 30.

+ BEAUSOLEIL, Claude, Claude ROBITAILLE et André ROY, "Entretien avec Michel Beaulieu», Hobo-Québec, nos 12-13, décembre 1973, p. 19-25.

+ RIBEYRON, Marie-Michèle, «Nos écrivains au travail», Perspectives, 31 août 1974, p. 12-16 (surtout p. 14).

+ [ANONYME], «Rencontre avec Michel Beaulieu, écrivain québécois», Journal de Genève, 14 novembre 1979, p. 17.

+ LARUE-LANGLOIS, Jacques, «ici et là... Michel Beaulieu », Le Livre d'ici, vol. V, no 46, 20 août 1980.

+ FILIATRAUlT, Jean, Michel Beaulieu: 27 mai 1980, Montréal, Service des transcriptions et dérivés de la radio, Maison de Radio-Canada, coll. «Portraits d'écrivains québécois», cahier no 30, 1980, 15 p.

+ ROYER, Jean, «Michel Beaulieu: fasciné par le futur», Le Devoir, 31 janvier 1981, p. 19.

+ GIGUÈRE, Richard, et Robert YERGEAU, «L'écriture doit être impudique», Lettres québécoises, no 30, été 1983, p. 46-54.

\section{11. Traductions $\mathrm{d}^{\prime} \propto u$ ves de Michel Beaulieu}

\section{I . 11 . 1. Liv res}

+ Spells of Fury, traduction d'Arlette Francière, Toronto, Exile Editions, 1984, 79 p.

+ Countenances, traduction de Josée Michaud, Toronto, Exile Editions, 1986, 121 p.

+ Kaleidoscope. Perils of a Solemn Body, traduction d'Arlette Francière, Toronto, Exile Editions, 1988, $92 \mathrm{p}$.

\section{I.11.2. Poèmes (publiés dans des volumes, des revues ou des journaux )}

+ «Écritures en traduction», traduction en italien par Angelo Bellettato, Dimensioni, vol. XVI, $\mathrm{n}^{\text {os }} 1-2$, avril 1972, p. 56.

+ "The Charms of Fury ", "Water and Blood of Bones », "Appointed Day or Letter From the Seasons IV (extraits)", traduction d'Arlette Francière, Contemporary Literature in Translation, vol. XXI, nos 14-17, été 1975, 40 p.

+ «Poems From Spells of Fury», traduction d'Arlette Francière, Exile, vol. VIII, nos 1-2, 1981, p. 180-186.

+ «Singele si apa Daselor», «Spuse zilei sau scrisori de sezon», traduction en roumain par Virgil Teodorescu et Petronela Negosanu, Steana Marilon Lacuri, «Poetri Canadieni Da Lamba Franceza», n 45, Bucarest, Editura Univers, 1981, p. 183-185.

+ «Tournoi de traduction», Ellipse, n ${ }^{\text {os }} 29-30,1982,170$ p.

+ "Two Poems From Charmes de la fureur », traduction d'Arlette Francière, Matrix, nº 15, printemps-été 1982, p. 71. 
+ «The Blues (poems)», traduction de Barry Callaghan, Exile, vol. X, n 2, 1985, p. 76-77.

+ «Il fallait aller beaucoup plus loin que le jour», traduction d'Arlette Francière, The Capilano Review, nº 35, 1985, p. 1.

+ «Poems From Snow», traduction de Josée Michaud, Exile, vol. X, nºs 3-4, 1985, p. 204-216.

\section{I.12. Traductions de Michel Beaulieu}

I.12.1. Ouvrages (Seules les traductions littéraires ont été recensé es.)

+ IBERVILLE-MOREAU (d'), Luc, Montréal perdu (Montréal Lost), Montréal, Quinze, 1977, 184 p.

+ ROBERTSON, Heather, Beauté tragique (A Terrible Beauty), traduit par Carol Dunlop-Hébert et Michel Beaulieu (poèmes), Montréal, Quinze, 1978, 240 p.

+ DOERKSON, Margaret, Jazzy, Montréal, Quinze, coll. «Prose étrangère», 1981, 271 p.

+ SCHWARZ, Herbert, Hommage à Picasso, 1881-1973 (Hommage to Pablo Picasso, 1881-1973), Québec, Éditions Isabeau, 1984, [n.p.].

\section{I . 12.2 . T h é ât r e}

+ WESKER, Arnold, Des frites, des frites, des frites... (Chips for Everything), présenté à Montréal par la Compagnie Jean Duceppe du 19 janvier au 25 février 1977.

+ LEONARD, Hugh, P'pa (Da), présenté à Montréal par la Compagnie Jean Duceppe du $1^{\text {er }}$ novembre au 2 décembre 1978.

+ LEWIN, Ira, Piège à rebours (DeathTrap), présenté à Montréal par la Compagnie Jean Duceppe du 28 mars au 28 avril 1979.

\section{12.3 . Po ésie}

+ GIBSON, Graham, «Mouvement perpétuel» («Perpetual Motion»), La Nouvelle Barre du jour, nº 126, mai 1983, p. 135-138.

+ "Poèmes traduits par Michel Beaulieu », Ellipse, no 36, 1986, p. 22-53. Traduction des poèmes «Fugue» («Fugue») de Robyn SARAH; «Le jeu du berceau» («Cat's Cradler»), «Dans les pièces d'hiver» («In Winter Rooms»), «Le cycliste retrouve sa cadence » («The Cyclist Recovers His Cadence»), «Voir» («To See») de Sharon THESEN; «Le, la, les» («The»), «Ceci, cela» («This \& That»), «Deux oies» («Two Geese») de Marilyn BOWERING.

\section{I.12.4. Pièce radiophonique (texte inédit)}

+ SAUNDERS, James, «La prochaine fois que je vous le chanterai», Théâtre actuel, Montréal, Radio-Canada, 14 février 1977.

\section{Bibliographie critique}

II.1. Livres, chapitres de livres, mémoire et thèses

+ MOISAN, Clément, Poésie des frontières, Montréal, Éditions Hurtubise/HMH, coll. «Constantes», 1979, 346 p.

+ ROYER, Jean, "Pour un avenir», Écrivains contemporains. Entretiens 2: 1977-1980, Montréal, l'Hexagone, 1981, p. 92-97.

+ BEAUSOLEIL, Claude, Les livres parlent, Trois-Rivières, Écrits des Forges, coll. «Estacades», 1984, p. 46-50. 
+ CLOUTIER, Guy, «Dans l'espoir que rien ne demeure» (repris de La Nouvelle Barre du jour, no 86, janvier 1980, p. 77-80) et «Lettre à Michel Beaulieu», Entrée en matière(s), Montréal, l'Hexagone, coll. «Essais littéraires», 1988, p. 51-55 et p. 56-57.

+ DUPRÉ, Louise, «Entre autres villes: Montréal», Lire Montréal, Montréal, Département d'études françaises, Université de Montréal, 1989, p. 73-84.

+ BONENFANT, Joseph, "Michel Beaulieu ou la familiarité de l'écriture», Passions du poétique, Montréal, l'Hexagone, coll. «Essais littéraires», 1992, p. 171-179. (Paru dans Ellipse, n 36, 1986, p. 4-12.)

+ LEMAY-BORÓWKA, Natasha Yvonne, "Michel Beaulieu: en(-)quête de l'immortalité», mémoire de maîtrise, Edmonton, University of Alberta, 1993, 115 p.

+ GERVAIS, André, " "Qui passe sa mort en vacances”», Sas, Montréal, Triptyque, 1994, p. 239243.

+ MIRON, Isabelle, «La quête de sens par le corps chez Michel Beaulieu et Juan Garcia», thèse de doctorat, Montréal, Université de Montréal, 2004, 255 f.

+ BISSONNETTE, Thierry, «Dynamiques du recueil de poésie chez trois poètes du Noroît: Alexis Lefrançois, Michel Beaulieu, Jacques Brault», thèse de doctorat, Québec, Université Laval, 2005, $353 \mathrm{f}$.

+ MAILHOT, Laurent et Pierre NEPVEU, La poésie québécoise. Des origines à nos jours, Montréal, Typo, coll. «Anthologie», 2007, 754 p.

\section{I. 2. A r ticles généraux}

+ BEAUCHAMP, Germain, «Poésie-3... mon œil!», Le Devoir, «supplément littéraire», 14 novembre 1970, p. V.

+ BEAUCHAMP, Germain, «Tout dire... peut-être», Le Devoir, $1^{\text {er }}$ mai 1971, p. 12.

+ BAUDOUIN, Monique, "Et on nomme cela "littérature"», Le Droit, 7 avril 1973, p. 15.

+ STRARAM, Patrick, «Lettre personnelle à Michel Beaulieu», Le Livre d'ici, vol. VI, nº 14, 7 janvier 1981, [n.p.].

+ ARCHAMBAULT, Claude, "Un homme entier et passionné», La Presse, 20 juillet 1985, p. C-3.

+ NEPVEU, Pierre, «Michel Beaulieu. Un engagement à la durée», Le Devoir, 20 juillet 1985, p. 1, 17, 20.

+ ROYER, Jean, «Vivre en poésie», Le Devoir, 20 juillet 1985, p. 17-20.

+ TOUPIN, Gilles, «Une œuvre inscrite dans le vécu quotidien», La Presse, 20 juillet 1985, p. C-3.

+ CLOUTIER, Guy, "Michel Beaulieu », Le Soleil, 23 juillet 1985, p. B-4.

+ BEAULIEU, Victor-Lévy, «Les fraises sauvages», Le Devoir, 27 juillet 1985, p. 17.

+ BÉLANGER, Paul, «Hommage à Michel Beaulieu», La Presse, 27 août 1985, p. A-7.

+ VAN SCHENDEL, Michel, «Métier et honneur à Michel Beaulieu », Le Devoir, 7 septembre 1985, p. 25.

+ MARTEL, Réginald, «Des lauriers pour le poète disparu », La Presse, 16 septembre 1985, p. B-5.

+ LECLERC, Rachel, «Michel Beaulieu. Le dernier aléa d'un précurseur », Lettres québécoises, no 39, automne 1985, p. 12-13.

+ CHAMBERLAND, Roger, "Michel Beaulieu et la scénographie du réel», Québec français, nº 60, décembre 1985, p. 26-27.

+ DAVID, Gilbert, "Décès de Michel Beaulieu, cofondateur de "Jeu" », Cahiers de théâtre Jeu, vol. II, no 35, 1985, p. 183.

+ PARIZEAU, Alice, «Michel Beaulieu. La mort d'un poète», La Presse, 24 mars 1986, p. B-10. 
+ FRANCIÈRE, Arlette, «Working with Michel/Après-midi avec Michel », Ellipse, n 36, 1986, p. 55-59.

+ MARLATT, Daphné, «Remembering Michel in English/Souvenirs anglais de Michel», Ellipse, $\mathrm{n}^{\circ}$ 36, 1986, p. 68-71.

+ MAY, C.R.P., "A Visit to Birmingham/Visite à Birmingham», Ellipse, n 36, 1986, p. 72-73.

+ MELANÇON, Robert, "Ordonner le chaos/The Regulation of Chaos», Ellipse, n 36, 1986, p. 64-67.

+ SARAH, Robyn, "A Love Affaire with Words/Une passion pour les mots», Ellipse, n 36, 1986, p. 60-63.

+ MELANÇON, Robert, «La deuxième personne du singulier», Estuaire, n 38, 1986, p. 141-154.

+ CLOUTIER, Guy, «Michel Beaulieu (1941-1985), collectionneur de fureurs, aurait aujourd'hui 50 ans ", Nuit blanche, ${ }^{\circ} 45$, septembre-novembre 1991, p. 8-9.

+ NEPVEU, Pierre, «La dure syntaxe de la conscience», dans Michel Beaulieu, Fuseaux. Poèmes choisis, Montréal, Éditions du Noroît, coll. «Ovale», 1996, p. 9-14.

+ BÉLANGER, Paul, «Hommage à Michel Beaulieu », Liberté, n 253, septembre 2001, p. 3-4.

+ CLOUTIER, Guy, «Le contemporain capital», Liberté, n 253, septembre 2001, p. 20-23.

+ FILION, Pierre, "Au coeur de la quadrature», Liberté, n 253, septembre 2001, p. 24-26.

+ LEBLANC, Gérald, «Kaléidoscope mnémonique à la mémoire de Michel Beaulieu », Liberté, $n^{\circ}$ 253, septembre 2001, p. 37-39.

+ MIRON, Isabelle, «Michel Beaulieu : l'autre voix», Liberté, n 253, septembre 2001, p. 43-45.

+ CARON, Pascal, «L'obsession du corps et la poésie québécoise. Une tentative d'ouverture théorique à partir des poèmes de Claude Beausoleil, Nicole Brossard et Michel Beaulieu », Études littéraires, vol. XXXVIII, nº 1, automne 2006, p. 91-106.

\section{II.3. Articles consacrés à des ouvrages en particulier II. 3.1. Poésie \\ Pour chanter dans les chaînes}

+ LAROSE, Paul, «Pour chanter dans les chaînes», Le Quartier latin, «supplément», vol. XLVII, $\mathrm{n}^{\circ}$ 18, 12 novembre 1964, p. 3.

+ CARDINAL, Pierre, "Pour chanter dans les chaînes », Échos-vedettes, vol. II, n 43, 14 novembre 1964, p. 24.

+ SAVOIE, Claude, "Ā moi la parole... à moi les paraboles», Le Petit Journal, semaine du 20 décembre 1964, p. A-47.

+ ROBERT, Guy, "Pour chanter dans les chaînes", Livres et auteurs canadiens 1964, Montréal, Éditions Jumonville, 1965, p. 53.

+ MARCEL, Jean, «Prose et poésies», L'Action nationale, vol. LIV, nº 5, janvier 1965, p. 504-508.

+ LAROSE, Paul, «La production littéraire de l'automne 1964 », Le Quartier latin, «supplément», vol. XLVII, n 29, 21 janvier 1965, p. 3.

+ DUCLOS, Jocelyne-Robert, "Pour chanter dans les chaînes », La Rotonde, "supplément", 4 février 1965, p. 2.

+ SAVOIE, Claude, «Nouveaux noms de notre poésie», Le Petit Journal, 27 juin 1965, p. 32.

\section{Le pain quotidien}

+ MAJOR, André, «Le pain quotidien», Livres et auteurs canadiens 1965, p. 97.

+ PRÉFONTAINE, Yves, «Poésie pas morte!», Maintenant, n 42, juin 1965, p. 213.

+ MAJOR, André, «Cinq poètes à l'œuvre», Le Petit Journal, 4 juillet 1965, p. 24. 
+ O'NEIL, Jean, «Le pain quotidien de Michel Beaulieu», La Presse, 10 juillet 1965, p. 5.

+ VALIQUETTE, Bernard, «Poésie pas morte», Échos-vedettes, vol. III, n 32, 28 août 1965, p. 27.

+ SYLVESTRE, Guy, «La poésie C.F. - Gemma Tremblay et Michel Beaulieu», Le Devoir, 23 octobre 1965, p. 13.

+ SYLVESTRE, Guy, «La poésie», University of Toronto Quarterly, vol. XXXV, n 4, 1965-66, p. 503-509.

+ MAJOR, André, «Trois jeunes poètes», Livres et auteurs canadiens 1965, Montréal, Éditions Jumonville, 1966, p. 97.

+ POISSON, Roch, «Le premier Prince des Poètes, une anti-pièce et Beaulieu... », Photo-Journal, 10-17 août 1966, p. 57.

"Ballades... et satires", dans Trois

+ ÉTIENNE, Gérard-V., «Beaulieu : une évolution rapide», Le Quartier latin, «supplément», vol. XLVII, n 50, 6 avril 1965, p. 8.

+ SYLVESTRE, Guy, «Poésie et pensum», Le Devoir, 24 avril 1965, p. 12.

+ BOUQUINISTE (LE), «Pour rejoindre l'universel», La Patrie, 13 mai 1965, p. 19.

+ LAROCHE, Max, «Trois», Livres et auteurs canadiens 1965, Montréal, Éditions Jumonville, 1966, p. 94.

\section{Apatride (Gestes II)}

+ ROBERT, Guy, "Apatride de Michel Beaulieu », Livres et auteurs canadiens 1966, Montréal, Éditions Jumonville, 1967, p. 79.

\section{Érosions}

+ POISSON, Roch, «Le dictionnaire de la rentrée», Photo-Journal, 23 août 1967, p. 66.

+ LAPOINTE, Gatien, "Chez Michel Beaulieu, non pas évolution, mais révolution», Le Soleil, 23 mars 1968, p. 34.

+ [ANONYME], «Érosions», Fiches bibliographiques de littérature canadienne, vol. II, nº 8, avril 1968, [n.p.].

+ THÉBERGE, Jean-Yves, «Érosions», Le Canada français, vol. CVIII, n 48, 25 avril 1968, p. 30.

+ SYLVESTRE, Guy, «La poésie», University of Toronto Quarterly, vol. XXXVII, n 4, juillet 1968, p. $578-588$.

\section{Charmes de la fureur}

+ BÉLANGER, Yrénée, «Charmes de la fureur», Le Livre canadien, vol. I, nº 81, 1970, [n.p.].

+ BASILE, Jean, «La fureur de Michel Beaulieu», Le Devoir, 28 février 1970, p. 14.

+ PALLASCO-MORIN, Ernest, «Des fureurs d'Oreste à celles de Michel», Photo-Journal, 9-15 mars 1970, p. 12.

+ ROBERT, Françoy, "Charmes de la fureur de Michel Beaulieu», Sept-Jours, 14 mars 1970, p. 17.

+ ROBERT, Françoy, «Pour mémoire», Le Devoir, 21 mars 1970, p. 14.

+ PARADIS, Suzanne, "Michel Beaulieu: du jeu verbal au mouvement intérieur du poème», Le Soleil, 4 avril 1970, p. 51.

+ RICHER, Julia, «Deux poètes», L'Information médicale et paramédicale, 5 mai 1970, p. 63.

+ LACÔTE, René, «Gaston Puel, Michel Beaulieu, Charles Le Quintec», Les Lettres françaises, $n^{\circ} 1334,13-19$ mai 1970, p. 8. 
+ PILON, Jean-Guy, «Vers un nouveau romantisme?», Le Devoir, 16 mai 1970, p. 15.

+ TURGEON, Michèle, «La critikeuse», L'Illettré, été 1970, [n.p.].

+ DALLARD, Sylvie, "Les Charmes de la fureur de Michel Beaulieu», Livres et auteurs québécois 1970, Montréal, Éditions Jumonville, 1971, p. 137.

+ DIONNE, René, «Où va notre poésie?», Relations, n 357, février 1971, p. 57.

+ MAJOR, Jean-René, [Sans titre], University of Toronto Quarterly, vol. XL, nº 4, été 1971, p. 440.

+ ÉTIENNE, Gérard, «Michel Beaulieu un poète de classe», Progrès-Évangélin, 14 janvier 1972, p. 16 (sur Charmes de la fureur et Paysage).

+ NEPVEU, Pierre, «Le poème inachevé», Études françaises, vol. XI, nº 1, février 1975, p. 55-65 (sur Charmes de la fureur, Paysage et Variables).

\section{Paysage}

+ RICHMOND, John, "Montreal's Literary Scene Three Books Lauched on Promising Voyage», The Montreal Star, 16 avril 1971, p. 12.

+ THÉBERGE, Jean-Yves, «Les paysages de Michel Beaulieu », Le Canada français, 30 juin 1971, p. 44.

+ PARADIS, Suzanne, "Aux prises avec son thème essentiel: le temps », Le Soleil, 14 août 1971, p. 36.

+ PILON, Jean-Guy, "“Poésie pour tous" - Michel Beaulieu», Le Devoir, 3 novembre 1971, p. 12.

+ LACROIX, Pierre, "Paysage de Michel Beaulieu», Livres et auteurs québécois 1971, Montréal, Éditions Jumonville, 1972, p. 151.

+ LACROIX, Pierre, «Paysage de Michel Beaulieu», Le Livre canadien, vol. III, n² 2, janvier 1972, [n.p.].

+ ÉTIENNE, Gérard, «Michel Beaulieu un poète de classe», Progrès-Évangéline, 14 janvier 1972, p. 16 (sur Charmes de la fureur et Paysage).

+ TREMBLAY, Régis, «Poètes consommateurs pour consommateurs», Le Soleil, 25 novembre 1972, p. 58.

+ MARCOTTE, Gilles, «Le poète et ses mots», Études françaises, vol. IX, nº 1, février 1973, p. 87.

+ NEPVEU, Pierre, «Le poème inachevé », Études françaises, vol. XI, no 1, février 1975, p. 55-65 (sur Charmes de la fureur, Paysage et Variables).

\section{Pulsions}

+ JASMIN, Claude, «Michel Beaulieu publie toujours», Journal de Montréal, 31 août 1973, p. 29.

+ THÉBERGE, Jean-Yves, "Pulsions de Michel Beaulieu, jeu et facilité», Le Canada français, 26 septembre 1973, p. 90.

+ BEAUSOLEIL, Claude, "Notes sur Pulsions de Michel Beaulieu», Hobo-Québec, nos $12-13$, décembre 1973, p. 26.

+ DEMERS, Jeanne, "Michel Beaulieu. Variables, Pulsions», Livres et auteurs québécois 1973, Québec, P.U.L., 1974, p. 133-136.

+ PILON, Jean-Guy, «En douze poètes (et treize recueils) : la fin de l'année 1973 », Le Devoir, 5 janvier 1974, p. 13.

+ MARCOTTE, Gilles, "Les mots comme des choses», Études françaises, vol. X, no 2, mai 1974, p. 134-135 (sur Pulsions et Variables).

+ HÉBERT, François, «Beaulieu (Michel) - Pulsions», Le Livre canadien, vol. VI, nº 168, mai 1975, [n.p.]. 


\section{Variables}

+ VACHON, Georges-André, "Variables de Beaulieu: qu'est-ce que cela veut dire? », dans Michel Beaulieu, Variables, 1973, p. 93-99 (article paru dans Le Devoir, «supplément», 19 mai 1973, p. 6).

+ PIAZZA, François, "Poésie d'ici: y aurait-il un ressac?", Montréal-Matin, 3 juin 1973, p. 16.

+ THÉBERGE, Jean-Yves, "Éclisses de la mémoire vous voletez bien bas - Michel Beaulieu », Le Canada français, 20 juin 1973, p. 86.

+ DEMERS, Jeanne, "Michel Beaulieu. Variables, Pulsions», Livres et auteurs québécois 1973, Québec, P.U.L., 1974, p. 133-136.

+ MARCOTTE, Gilles, "Les mots comme des choses», Études françaises, vol. X, n 2, mai 1974, p. 134-135 (sur Pulsions et Variables).

+ NEPVEU, Pierre, «Le poème inachevé», Études françaises, vol. XI, nº 1, février 1975, p. 55-65 (sur Charmes de la fureur, Paysage et Variables).

\section{FM. Lettres des saisons III}

+ LAROCQUE, Gilbert, "Michel Beaulieu livre des lettres oubliées jusqu'ici dans un tiroir», Le Livre d'ici, vol. I, nº 20, 1976, [n.p.].

+ BEAUSOLEIL, Claude, «FM. Lettres des saisons III», Le Jour, 27 février 1976, p. 31.

+ [ANONYME], «Trois recueils de poèmes: Griefs de Gilbert Langevin, FM de Michel Beaulieu et La mort était extravagante de Geneviève Amyot», Ici-Radio, 17 avril 1976, n² 206, p. 12.

+ DIONNE, René, «FM. Lettres des saisons III», University of Toronto Quarterly, vol. XLV, nº 4, été 1976, p. 348.

+ JANOIX, Georgette, «FM. Lettres des saisons III», Le Livre canadien, vol. VII, n² 254, septembre 1976, [n.p.].

+ BONENFANT, Joseph, «Michel Beaulieu, FM. Lettres des saisons III», Livres et auteurs québécois 1976, P.U.L., 1977, p. 167-168.

\section{Anecdotes}

+ RIVARD, Yvon, "La profondeur poétique de Michel Beaulieu », Le Livre d'ici, vol. II, nº 37, 22 juin 1977, [n.p.].

+ GEMME, Gilles, «Beaulieu s'est fait fondeur», Le Canada français, 22 juin 1977, p. 32.

+ BEAUSOLEIL, Claude, «Livres (Anecdotes)», Québec Rock, juillet 1977, p. 28.

+ HÉBERT, François, «Anecdotes de Michel Beaulieu », Le Jour, 29 juillet au 4 août 1977, p. 25.

+ GIRARD, Michel, "Anecdotes», Nos livres, vol. VIII, n 283, octobre 1977, [n.p.].

+ MOISAN, Clément, «Nouveautés (Anecdotes - Éd. Du Noroît)», Québec français, octobre 1977, p. 11.

+ RENAUD, Jacques, «Douze poètes québécois. Les bons sentiments ne font pas la poésie... les mauvais non plus», Le Devoir, 22 octobre 1977, p. 34.

+ HÉBERT, François, «LeFrançois, Beaulieu, Nepveu, Vanier», Liberté, vol. XIX, no 114, novembre-décembre 1977, p. 95-97 (sur Anecdotes et L'octobre).

+ MALENFANT, Paul-Chanel, «Michel Beaulieu, Anecdotes, L'octobre, suivi de Dérives, Le cercle de justice», Livres et auteurs québécois 1977, Québec, P.U.L., 1978, p. 160-164.

\section{Le cercle de justice}

+ MALENFANT, Paul-Chanel, "Michel Beaulieu, Anecdotes, L'octobre, suivi de Dérives, Le cercle de justice», Livres et auteurs québécois 1977, Québec, P.U.L., 1978, p. 160-164. 
+ PARADIS, Suzanne, "La saison plénière de Michel Beaulieu », Le Devoir, 18 mars 1978, p. 35 (sur Le cercle de justice, Indicatif présent et L'octobre).

+ BOURASSA, André-G., "Justice et Beaulieu», Lettres québécoises, n 10, avril 1978, p. 35 (survol rétrospectif de l'œuvre jusqu'au Cercle de justice).

+ GAULIN, André, "L'octobre, suivi de Dérives - Le cercle de justice», Québec français, nº 30, mai 1978, p. 9.

+ ROYER, Jean, «Livres. Le cercle de justice», Montréal-Matin, 19 mai 1978, p. 25.

+ HAMEL, Marie-Andrée, «Michel Beaulieu. Une œuvre poétique importante et rigoureuse dans son ensemble », Le Livre d'ici, vol. IV, n² 2, 18 octobre 1978, [n.p.]. (Sur Le cercle de justice et L'octobre, suivi de Dérives.)

\section{Indicatif présent}

+ PARADIS, Suzanne, «La saison plénière de Michel Beaulieu», Le Devoir, 18 mars 1978, p. 35 (sur Le cercle de justice, Indicatif présent et L'octobre).

+ BEAUSOLEIL, Claude, «Indicatif présent», La Nouvelle Barre du jour, nº 66, mai 1978, p. 74-75.

\section{L'octobre, suivi de Dérives}

+ HÉBERT, François, «LeFrançois, Beaulieu, Nepveu, Vanier», Liberté, vol. XIX, nº 114, novembre-décembre 1977, p. 95-97 (sur Anecdotes et L'octobre).

+ MALENFANT, Paul-Chanel, "Michel Beaulieu, Anecdotes, L'octobre, suivi de Dérives, Le cercle de justice», Livres et auteurs québécois 1977, Québec, P.U.L., 1978, p. 160-164.

+ PARADIS, Suzanne, «La saison plénière de Michel Beaulieu », Le Devoir, 18 mars 1978, p. 35 (sur Le cercle de justice, Indicatif présent et L'octobre).

+ GAULIN, André, "L'octobre, suivi de Dérives - Le cercle de justice», Québec français, n 30, mai 1978, p. 9.

+ HAMEL, Marie-Andrée, «Michel Beaulieu. Une œuvre poétique importante et rigoureuse dans son ensemble », Le Livre d'ici, vol. IV, n 2, 18 octobre 1978, [n.p.]. (Sur Le cercle de justice et L'octobre, suivi de Dérives.)

\section{Comment ça va?}

+ BEAUSOLEIL, Claude, «Des textes quotidiens : Baudrillard, Beaulieu», Le Devoir, 24 février 1979, p. 26.

\section{Oracle des ombres}

+ MELANÇON, Robert, «Gagner sur le silence», Le Devoir, 25 août 1979, p. 15.

+ TREMBLAY, Régis, «Littérature québécoise. Oracle des ombres. Michel Beaulieu. Poésie», Le Soleil, 27 septembre 1979, p. B-11.

+ GAUDET, Gérald, "Michel Beaulieu. Oracle des ombres », Livres et auteurs québécois 1979, Québec, P.U.L., 1980, p. 94-95.

+ BEAUSOLEIL, Claude, "Sur cet Oracle des ombres suivi de Un texte épars", La Nouvelle Barre du jour, no 86, janvier 1980, p. 75-76.

+ BÉLANGER, Marcel, «Michel Beaulieu. L'Oracle», Le Livre d'ici, vol. V, nº 21, 27 février 1980, [n.p.].

+ D’ALFONSO, Antonio, "Oracle des ombres», Nos livres, vol. XII, n² 263, juin-juillet 1981, [n.p.]. 


\section{Desseins}

+ DE BELLEFEUILLE, Normand, «La tentation de l'Histoire», Spirale, n 10, juin 1980, p. 11.

+ ClOUTIER, Guy, «Desseins», Le Bulletin Pantoute, nº 2, juin-août 1980, p. 6.

+ NEPVEU, Pierre, «La poésie I. De l'“importance" de la littérature», Lettres québécoises, n 19 , automne 1980, p. 28-31.

+ DIONNE, André, «Desseins », Nos livres, vol. XI, n 320, novembre 1980, [n.p.].

+ DIONNE, René, «Michel Beaulieu, Desseins... », Le Droit, 15 novembre 1980, p. 16.

+ GAULIN, André, "Nouveautés. Poésie», Québec français, n 40, décembre 1980, p. 15.

+ PARADIS, Suzanne, "Michel Beaulieu. Desseins», Livres et auteurs québécois 1980, Québec, P.U.L., 1981, p. 86-88.

+ [ANONYME], «Avant de refermer la garde-robe de l'oubli», Le Canada français, 7 janvier 1981, p. 68.

+ BEAUSOLEIL, Claude, «Michel Beaulieu: écrire la suite», Le Devoir, 31 janvier 1981, p. 21.

+ TREMBLAY, Régis, "Michel Beaulieu écrit pour le plaisir d'avoir écrit», Le Soleil, 14 mars 1981, p. D-10.

\section{Visages}

+ BEAUSOLEIL, Claude, «Visages intérieurs», Le Devoir, 5 décembre 1981, p. 21.

+ THÉRIAULT, Jacques, «Michel Beaulieu. Vingt-cinq années d'expériences amoureuses avec l'acte poétique», Le Livre d'ici, vol. VII, nº 16, 20 janvier 1982, [n.p.].

+ BEAUliEU, Ivanhoé, «Beaulieu: le désir poursuivi», La Presse, 13 février 1982, p. C-3.

+ CHAMBERLAND, Roger, «Visages», Québec français, n 45, mars 1982, p. 16.

+ THOMAS, Réjean, «Visages», Nos livres, vol. XIII, no 98, mars 1982, [n.p.].

+ MELANÇON, Robert, «Michel Beaulieu, Jean-Pierre Issenhuth», Liberté, vol. III, no 3 (n 141), mai-juin 1982, p. 118-120.

+ BOUCHARD, Christian, "Michel Beaulieu - Visages», Estuaire, n² 25, automne 1982, p. 86-87.

+ LAWALL, Sarah, "Visages», French Review, vol. LVII, n 1, octobre 1983, p. 130-131.

\section{Kaléidoscope ou Les aléas du corps grave}

+ ROYER, Jean, «La poésie qui se fréquente», Le Devoir, 15 décembre 1984, p. 27.

+ DESGENT, Jean-Marc, "Michel Beaulieu. Kaléidoscope ou Les aléas du corps grave», Nos livres, janvier 1985, p. 16-17.

+ TOUPIN, Gilles, «Poésie d'ici. Michel Beaulieu ou la vie lumineuse», La Presse, 9 février 1985, p. E-2.

+ CLOUTIER, Guy, «Michel Beaulieu ou la feinte indifférence», Nuit blanche, nº 17, févriermars 1985, p. 4.

+ BAYARD, Caroline, "Du retour des années 60 aux revers de l'amour », Lettres québécoises, $\mathrm{n}^{\circ} 37$, printemps 1985 , p. 50-52.

+ BROCHU, André, «Des fous et des autres", Voix et Images, vol. X, n 3, printemps 1985, p. 179-187.

+ PAQUIN, Jacques, «Michel Beaulieu. Kaléidoscope ou Les aléas du corps grave», Estuaire, nº 35, printemps 1985, p. 91.

+ MALENFANT, Paul-Chanel, "Kaléidoscope ou Les aléas du corps grave», Nuit blanche, n 18 , avril-mai 1985, p. 5-6.

+ BEAUSOLEIL, Claude, «Michel Beaulieu. Kaléidoscope ou Les aléas du corps grave», Vice Versa, vol. II, nº 4, juin-juillet 1985, p. 19. 
+ NEPVEU, Pierre, «Les matières du réel», Spirale, n 53, juin 1985, p. 6.

+ ROYER, Jean, «Les narratifs du monde enchevêtré», Le Devoir, 8 juin 1985, p. 23.

+ GILBERT LEWIS, Paula, "Kaléidoscope ou Les aléas du corps grave», The French Review, vol LX, n² 2, décembre 1986, p. 287-288.

\section{$\mathrm{Vu}$}

+ ROYER, Jean, «Poésie», Le Devoir, 2 décembre 1989, p. D-3.

+ COPPENS, Patrick, «Michel Beaulieu, ramoneur du silence», Vu, Montréal/Pantin, Éditions du Noroît/Castor astral, 1989, p. IX-XII.

+ NOËL, Bernard, «M.B.», Vu, Montréal, Éditions du Noroît; Pantin (France), Castor astral, 1989, p. XII-XVI.

+ CORRIVEAU, Hugues, «La poésie est une extravagance», Lettres québécoises, nº 58, été 1990, p. 35-36.

\section{Indicatif présent et autres poèmes}

+ [ANONYME], «Michel Beaulieu redécouvert», Le Soleil, 21 février 1994, p. B-7.

\section{Fuseaux}

+ BERTIN, Raymond, «Fuseaux - Poèmes choisis», Voir, vol. XXI, n 10, 1 ${ }^{\text {er }}$ août 1996, p. 25.

\section{Trivialités}

+ CLOUTIER, Guy, [Préface], Trivialités, Montréal, Éditions du Noroît, 2001, [n.p.].

+ CANTIN, David, «La vie comme une révélation», Le Devoir, 2 février 2002, p. D-3.

+ BEAUSOLEIL, Claude, «Michel Beaulieu: la voix du poète», La Presse, 17 mars 2002, p. B-4.

+ LANDRY, Gabriel, «Du trivial au sublime», Voix et Images, vol. XVII, n 3, printemps 2002, p. 576-579.

+ MIRON, Isabelle, «L'excentrique Beaulieu», Liberté, n 258, novembre 2002, p. 196-201.

\section{I . 3 . 2. R o m a n}

Je tourne en rond mais c'est autour de toi

+ POISSON, Roch, "Michel Beaulieu tourne en rond mais c'est autour d'elles », Photo-Journal, 3-10 septembre 1969, p. 42.

+ MARTEL, Réginald, «Un jeu solitaire et stérile», La Presse, 6 septembre 1969, p. 29.

+ MAJOR, André, "Quand tourner en rond devient un art abusif», Le Devoir, 13 septembre 1969, p. 13.

+ FERRON, Jacques, «Le thème des grilles », Le Petit Journal, 14 septembre 1969, p. 81.

+ PALLASCIO-MORIN, Ernest, "Ce que j'en pense», Photo-Journal, 24 septembre-1er octobre 1969, p. 21.

+ MAILHOT, François, «Voulez-vous tourner en rond?», Le Soleil, 18 octobre 1969, p. 32.

+ SAINT-ONGE, Paule, «D'un jeune romancier à Napoléon», Châtelaine, vol. X, nº 11, novembre 1969, p. 46.

+ BÉLANGER, Yrénée, "Je tourne en rond mais c'est autour de toi de Michel Beaulieu», Livres et auteurs québécois 1969, Montréal, Éditions Jumonville, 1970, p. 41.

+ BÉLANGER, Yrénée, «Je tourne en rond mais c'est autour de toi », Le Livre canadien, vol. I, n 2, 1970, [n.p.]. 
+ ROBERGE, Francyne, «Les jeunes romanciers: moisson clairsemée», Le Devoir, 18 avril 1970, p. 18.

+ ROBIDOUX, Réjean, «Romans, récits, nouvelles, contes», University of Toronto Quarterly, vol. XXXIX, n 4, juillet 1970, p. 294-459.

+ BEAUDET, André, "Posface. Circonvolutions verbales», dans Michel Beaulieu, Je tourne en rond mais c'est autour de toi, 1980, p. 184-191. (Repris dans André Beaudet, Interventions du parlogue 2, Montréal, Les Herbes rouges, $n^{\text {os }} 166-167,1988$, p. 55-58.)

\section{La représentation}

+ GODIN, Jean-Cléo, "Le roman au nom du père, des petits-fils et des grands-pères », Études françaises, vol. VIII, n 4, novembre 1972, p. 438-439.

+ GODIN, Jean-Cléo, «La représentation», Le Livre canadien, vol. III, n² 247, novembre 1972, [n.p.].

+ VIGNEAULT, Robert, "La représentation de Michel Beaulieu », Livres et auteurs québécois 1972, Montréal, Éditions Jumonville, 1973, p. 56-59.

+ ARCHAMBAULT, Gilles, «Lire au soleil... ou au coin du feu», Le Devoir, «supplément littéraire», 19 mai 1973, p. 13.

+ CLOUTIER, Guy, «Postface critique», dans Michel Beaulieu, La représentation, Montréal, Quinze, coll. «Présence», 1980, p. 201-203.

\section{Sylvie Stone}

+ RICARD, François, «Sylvie Stone de Michel Beaulieu», Le Jour, vol. I, n 38, 13 avril 1974, p. V-2.

+ THÉRIAULT, Jacques, «Pour saluer Michel Beaulieu et évoquer sa Sylvie Stone», Le Devoir, 17 avril 1974, p. 12.

+ THÉRIAUlT, Jacques, «Sylvie Stone», Québec-Presse, 28 avril 1974, p. 30.

+ MARTEL, Réginald, «L'amour, mais pas toujours», La Presse, 11 mai 1974, p. E-3.

+ TREMBLAY, Robert, «Sylvie Stone de Michel Beaulieu: un anti-personnage obsédant», Le Soleil, 18 mai 1974, p. 72.

+ PALLASCIO-MORIN, Ernest, «Roman qui se fait», Photo-Journal, 10-16 juin 1974, p. 16.

+ ÉTHIER-BLAIS, Jean, «Le dernier (Michel) Beaulieu», Le Devoir, 22 juin 1974, p. 15.

+ HURTEAU, Laure, «Les éditions canadiennes. Sylvie Stone», L’Information médicale, octobre 1974, p. 55.

+ SAINT-ONGE, Paule, «Lectures - Sylvie Stone», Châtelaine, vol. XV, nº 10, octobre 1974, p. 90-91.

+ SAINT-ONGE, Paule, «Sylvie Stone», Le Livre canadien, vol. V, n 266, octobre 1974, [n.p.].

+ GAY, Paul, «Sylvie Stone de Michel Beaulieu», Le Droit, 12 octobre 1974, p. 20.

+ HATHORN, Ramora, «Sylvie Stone», Journal of Canadian Fiction, vol. III, no 4, 1975, p. 92-93.

+ HÉBERT, François, "Michel Beaulieu, Sylvie Stone», Livres et auteurs québécois 1974, Québec, P.U.L., 1975, p. 80-82.

+ PELLETIER, Jacques, «Sylvie Stone», Livres et auteurs québécois 1974, Québec, P.U.L., 1975, p. 16-17.

+ HÉBERT, François, «Le noir et le blanc, le bleu et le rouge », Études françaises, vol. XI, nº 2 , mai 1975, p. 111-119.

+ POULIN, Gabrielle et René DIONNE, «Sylvie Stone», University of Toronto Quarterly, vol. XLIV, no 4, été 1975, p. 320. 
+ POUPART, Jean-Marie, "Postface. Quelques voies de service», dans Michel Beaulieu, Sylvie Stone, Montréal, Quinze, coll. «Présence», 1980, p. 181-183.

\section{I . 3 . 3. R éc it}

$X$

+ PILON, Jean-Guy, «X de Michel Beaulieu», Digeste Éclair, vol. V, n 10, octobre 1968, p. 23.

\section{I 3 . 4. Monographie}

P. V. Beaulieu

+ DE ROUSSAN, Jacques, «L'œil sur P. V. Beaulieu», Le Livre d'ici, vol. VII, n 7, 18 novembre 1981, [n.p.].

\section{I . 3 . 5. T h é ât r e}

Jeudi soir en pleine face

+ TALBOT, Michelle, «De "La Manufacture" au Quat-sous... pour être son propre patron», Dimanche-Matin, 18 janvier 1976, p. B-11.

+ BERNATCHEZ, Raymond, «Deux filles qui se regardent un jeudi soir en pleine face», MontréalMatin, 24 janvier 1976, p. 20.

+ VALLIÈRES, Pierre, «Jeudi soir en pleine face, deux filles se vident le cœur», Le Jour, 28 janvier 1976, p. 24.

+ VALLIÈRES, Pierre, «Mille regrets, des promesses... Deux filles à perte de parole... », La Presse, 28 janvier 1976, page $\mathrm{H}-1$.

+ BROUSSEAU, Jean-Pierre, "Jeudi soir en plein face: succès d'estime ", La Presse, 30 janvier 1976, p. B-10.

+ DUMAS, Carmel, «Play Brings You Near Loves, Fears», The Gazette, 30 janvier 1976, p. 33.

+ SABBATH, Lawrence, "At Theatre de Quat'sous. Two-Women Play Shows Insight», The Montreal Star, 30 janvier 1976, p. B-8.

+ BERNATCHEZ, Raymond, «J'parle pour parler», Montréal-Matin, 31 janvier 1976, p. 17.

+ GRUSLIN, Adrien, "Nouvelle troupe pour nouvelle pièce», Le Devoir, 31 janvier 1976, p. 14.

+ PERREAULT, Pascale, «On y joue», Le Journal de Montréal, «supplément du samedi», 31 janvier 1976, p. 20.

+ GRUSLIN, Adrien, «Un jeudi soir beaucoup trop ordinaire», Le Devoir, 5 février 1976, p. 15.

+ TALBOT, Michelle, "Jeudi soir en pleine face», Dimanche-Matin, 15 février 1976, p. B-10.

+ VILLEMAIRE, Yolande, "Jeudi soir en pleine face», Cahiers de théâtre Jeu, nº 2, printemps 1976, p. 98-99. 